\title{
Singular Velocities of Even-Rank Affine Distributions
}

\author{
Marek Rupniewski
}

Received: 28 June 2010 / Revised: 16 September 2014 / Published online: 12 November 2014

(C) The Author(s) 2014. This article is published with open access at Springerlink.com

\begin{abstract}
Singular curves, which are projections of singular extremals, play a special role in control theory and the theory of distributions. In this paper, we show that singular velocities, tangent to singular curves, determine affine distributions that are simultaneously of an evenrank and co-rank 2 . If $D$ is such a distribution, then its singular velocities span a distribution $C^{D}$ (of a rank two times smaller than that of $D$ ) that, together with its Lie square, forms the initial distribution, i.e.,

$$
D=C^{D}+\left[C^{D}, C^{D}\right]
$$

We also provide canonical constructions of vector fields that are $C^{D}$ generators, canonical differential forms that are $D$ cogenerators, and some functional invariants related to distribution of the considered type.
\end{abstract}

Keywords Affine distributions · Singular extremals · Singular curves

Mathematics Subject Classification (2010) 93B99 · (55R10 · 53B99)

\section{Introduction}

A rank $l$ affine distribution $D$ on a smooth $n$-dimensional manifold $M$ is a subbundle of the tangent bundle $T M$ such that locally, around any $q \in M$, there exist smooth and linearly independent vector fields $f_{0}, f_{1}, \ldots, f_{l}$, called generators of $D$, such that

$$
D=f_{0}+\operatorname{span}\left\{f_{1}, \ldots, f_{l}\right\} .
$$

The author was supported by Ministry of Research and Higher Education grant N20103932/2703.

M. Rupniewski $(\bowtie)$

Faculty of Electronics and Information Technology, Institute of Electronic Systems, Warsaw University of Technology, Nowowiejska 15/19, 00-665 Warsaw, Poland

e-mail: M.Rupniewski@ise.pw.edu.pl 
Thus, an affine distribution can be considered a control-affine system:

$$
\dot{q}=f_{0}(q)+\sum_{i=1}^{l} u_{i} f_{i}(q)
$$

seen from a geometric viewpoint. If the corank, $n-l$, of distribution $D$ is smaller than its rank, then it is usually more convenient to describe the distribution in terms of its affine cogenerators, i.e., linearly independent one-forms

$$
\omega_{0}, \omega_{1}, \ldots, \omega_{n-l-1},
$$

such that their inner products with every section of $D$ is identically one:

$$
\left.\forall v \in D_{q}: v\right\lrcorner \omega_{i}=1, \quad i=0,1, \ldots, n-l-1 .
$$

One of the main questions addressed by control theorists is that of the feedback equivalence of two control systems. From a geometric viewpoint, if restricted to the control-affine systems, this question may be stated as follows: "Are two given affine distributions diffeomorphic or not?". In low dimensions, there are methods that allow the answer to be worked out. These methods are based on the constructions of some complete sets of invariants. Often, these invariants are obtained studying the so-called singular curves, see, e.g., [1, $12,14,20]$. One of the most impressive results of this kind is presented in [2]. In terms of singular velocities (i.e., velocities of singular curves), and without going into detail, we could restate that result as follows. Generic affine distributions of rank 2 on a 4-dimensional manifold $M$ are completely determined by their singular velocities, which form rank 1 distributions that are in turn completely classified in the same paper [2].

Singular curves of affine distributions correspond to so-called singular extremals (see Section 2), which appear in the study of the time-optimal trajectories of control-affine systems (see, e.g., [3]). The fact that a singular extremal may be a non-trivial minimizer was first observed by Montgomery [16]. However, one must note that such phenomena are possible only for distributions of small rank (see [6] and [8] for the case of affine and vector distributions, respectively).

In [19], we showed that generic distributions from $\mathcal{A}(M, 2 k), \operatorname{dim} M>2 k+2$ (here and henceforth, $\mathcal{A}(M, l)$ stands for the class of rank $l$ distributions on a manifold $M)$ are also uniquely determined by their singular velocities. The distributions are affinely spanned by these velocities. This situation is quite analogous to the case of the odd-rank vector distributions of corank less than the dimension of the manifold minus 3, see [17]. Quite surprisingly, generic affine distributions of corank 2 are no longer spanned by their singular velocities, although they are still uniquely determined by them (see Theorem 2). This phenomenon is similar to that observed in the world of vector distribution and presented in [13].

Before stating a theorem that reveals relations between a corank 2 affine distribution $D$ and its singular velocities, let us note that such a distribution can be identified with its polarizer $D^{\top}$ that is a rank 1 affine subbundle of the cotangent bundle:

$$
\left.D_{q}^{\top}=\left\{\omega \in T_{q}^{*} M \mid D_{q}\right\lrcorner \omega=1\right\}
$$

Throughout the paper, we will denote the vector part of an affine distribution $D$ by $\underline{D}$, and its linear hull by $\bar{D}$, i.e., in the context of (1),

$$
\underline{D}=\operatorname{span}\left\{f_{1}, \ldots, f_{l}\right\} \subset \bar{D}=\operatorname{span}\left\{f_{0}, f_{1}, \ldots, f_{l}\right\} .
$$

Thus, for a distribution $D$ of $\operatorname{rank} 2 k, \underline{D}$ and $\bar{D}$ are the vector distributions of ranks $2 k$ and $2 k+1$, respectively. 
The fibers $D_{q}^{\top}$ have an affine-line structure and hence they define pencils of skewsymmetric forms:

$$
\begin{aligned}
& \underline{\mathrm{A}}_{q}^{*}: D_{q}^{\top} \rightarrow\left(\underline{D}_{q} \wedge \underline{D}_{q}\right)^{*}, \\
& \overline{\mathrm{A}}_{q}^{*}: D_{q}^{\top} \rightarrow\left(\bar{D}_{q} \wedge \bar{D}_{q}\right)^{*},
\end{aligned}
$$

where

$$
\left.\underline{\mathrm{A}}_{q}^{*}(p)(v \wedge w)=[X, Y](q)\right\lrcorner p, \quad p \in D_{q}^{\top}, v=X(q), w=Y(q)
$$

and $X, Y$ are germs of sections of distribution $\underline{D} . \overline{\mathrm{A}}_{q}^{*}$ is defined analogously: $\overline{\mathrm{A}}_{q}^{*}(p)$ is an extension of $\underline{\mathrm{A}}_{q}^{*}(p)$ from $\underline{D}_{q} \wedge \underline{D}_{q}$ to $\bar{D}_{q} \wedge \bar{D}_{q}$ such that if $v$ and $w$ are in $\underline{D}_{q}$ then vector fields $X$ and $Y$ in Eq. 2 are sections of $\underline{D}$, and if $v$ and $w$ are not in $\underline{D}_{q}$ then $X$ and $Y$ in the equation are sections of the affine subbundles $\alpha_{X} D$ and $\alpha_{Y} D$, where $\alpha_{X}$ and $\alpha_{Y}$ are constants such that

$$
\frac{1}{\alpha_{X}} v \in D_{q} \quad \text { and } \quad \frac{1}{\alpha_{Y}} w \in D_{q} .
$$

Equivalently, using the well-known formula,

$$
X\lrcorner Y\lrcorner d \omega=[X, Y]\lrcorner \omega+Y(X\lrcorner \omega)-X(Y\lrcorner \omega),
$$

one may define the above pencils evaluated at $p \in D_{q}^{\top}$ as

$$
v \wedge w \mapsto v\lrcorner w\lrcorner d \omega(q), \quad \omega \in D^{\top}, \omega(q)=p,
$$

where $v, w \in \underline{D}_{q}$ for $\underline{\mathrm{A}}^{*}$ and $v, w \in \bar{D}_{q}$ for $\overline{\mathrm{A}}^{*}$.

Let $\mathcal{A}_{q}(n, l)$ denotes the class of germs at $q \in \mathbb{R}^{n}$ of distributions $D \in \mathcal{A}\left(\mathbb{R}^{n}, l\right)$. According to classical theory of matrix pencils [9, chapter 12] and its applications to the case of skew-symmetric matrices [10, section 6], the following proposition holds.

Proposition 1 Let the pencil $\overline{\mathrm{A}}^{*}$ of a distribution $D \in \mathcal{A}_{q}(2 k+2,2 k)$ has the minimal Kronecker index equal $k$. Then for each $p \in D_{q}^{\top}$, the kernel $V_{p}$ of the form $\overline{\mathrm{A}}_{q}^{*}(p)$ is onedimensional. Moreover, for a fixed affine basis $\left(p_{0}, p_{1}\right)$ in $D_{q}^{\top}$, one may parametrize the set of all kernels $V_{p}, p \in D_{q}^{\top}$, by a $\bar{D}_{q}$-valued polynomial mapping:

$$
p=t p_{1}+(1-t) p_{0} \mapsto t^{k} v_{k}+t^{k-1} v_{k-1}+\cdots+t v_{1}+v_{0} \in V_{p},
$$

where the linearly independent vectors $v_{0}, \ldots, v_{k}$ span the fiber $L_{q}$ of a rank $k+1$ distribution $L \subset \bar{D}$. Conversely, if for each $p \in D_{q}^{\top}$, the kernel $V_{p}$ is one-dimensional and these kernels span a $(k+1)$-dimensional subspace in $\bar{D}_{q}$, then the pencil $\overline{\mathrm{A}}^{*}$ has minimal Kronecker index equal $k$.

With a help of distribution $L$ described above, one may define a rank $k$ affine distribution $C^{D} \subset D$ :

$$
C^{D}=L \cap D,
$$

provided that $L \not \subset \underline{D}$, which can be assured by regularity of pencil $\underline{A}_{q}^{*}$. The regularity means that $\underline{\mathrm{A}}_{q}^{*}(p)$ is non-singular for at least one $p \in D_{q}^{\top}$ or, using $D$ cogenerators $\omega_{0}, \omega_{1}$, that the polynomial mapping

$$
t \mapsto \omega_{0} \wedge \omega_{1} \wedge\left(t d \omega_{1}+(1-t) d \omega_{0}\right)^{k}(q)
$$

does not vanish identically. 
Theorem 2 (Local determinacy theorem) Let $D \in \mathcal{A}_{q}(2 k+2,2 k)$ satisfy the following two conditions:

$$
\underline{\mathrm{A}}_{q}^{*} \text { is a regular pencil, }
$$

$$
\overline{\mathrm{A}}_{q}^{*} \text { has minimal Kronecker index } k \text {, }
$$

then the set $S^{D}$ of singular velocities satisfy the following relations

$$
C^{D}=\operatorname{aff-span} S^{D} \in \mathcal{A}_{q}(2 k+2, k)
$$

and

$$
\left[C^{D}, C^{D}\right] \subset \underline{D}
$$

Moreover, if

$$
\operatorname{dim}\left(C_{q}^{D}+\left[C^{D}, C^{D}\right]_{q}\right)=2 k,
$$

then $C^{D}$ is a unique rank $k$ distribution satisfying

$$
C^{D}+\left[C^{D}, C^{D}\right]=D
$$

Conditions (G1)-(G3) are of the open type, and they can be simultaneously satisfied (see Section 4). Therefore, one may prove (see [19] for more detail) that a pair of linearly independent 1 -forms $\omega_{0}, \omega_{1}$ on a $(2 k+2)$-dimensional manifold $M$ affinely cogenerate distribution $D \in \mathcal{A}\left(M^{\prime}, 2 k\right)$ satisfying these conditions, where $M^{\prime}$ is an open and dense submanifold of $M$. Hence, on the basis of Theorem 2, one may claim that not only germs but also generic affine distributions of an even rank and corank 2 are completely determined by their singular velocities.

A germ $D \in \mathcal{A}_{q}(2 k+2,2 k)$ can be often determined by another set of curves. One may define a vector field $f_{0}$ as the unique intersection of $D$ and the characteristic line field [15] of $\bar{D}$. Then, $D$ is uniquely determined by the integral curves of $f_{0}$ and the singular curves of $\underline{D}[13]$. More precisely,

$$
D=f_{0}+\underline{D},
$$

where $\underline{D}$ is determined by its singular curves, provided that there are at least two of them passing through $q$ (for a generic distribution $D \in \mathcal{A}(M, 2 k), \operatorname{dim} M=2 k+2$, there may exist an open set through which no singular curve of $\underline{D}$ passes; see [13]).

In the context of Theorem 2, we must also mention the result of Bonnard [5], who proved that affine distributions are determined by singular extremals. Let us emphasize that Theorem 2 constitutes a result that is much stronger: it is enough to know singular curves, which are projections of singular extremals, to recover the distribution.

In this paper, we also reveal the structure of the bundle $S^{D}$ whose fiber $S_{q}^{D}$ can be treated as a rational normal curve on projectivization $\mathbb{P} C_{q}^{D}$, i.e., as a Veronese embedding of the projective line into $\mathbb{P} C_{q}^{D}$.

Theorem 3 If $D \in \mathcal{A}_{0}(2 k+2,2 k)$ satisfies Conditions (G1), (G2) and (G4)-(G6) (nondegeneracy Conditions (G4)-(G6) are formulated in Section 5), then there is a canonical way of defining the following objects:

(1) a system of cogenerators of $D$,

(2) a system $\left(\hat{f}_{0}, \hat{f}_{1}, \ldots, \hat{f}_{k}\right)$ of generators of $C^{D}$, 
(3) a system of $k-2$ real valued functions $a_{1}, \ldots, a_{k-2}$ such that fibers $S_{q}^{D}$ have parametrizations

$$
t \mapsto \hat{f}_{0}+\frac{1}{\widehat{R}_{q}(t)} \sum_{i=1}^{k} t^{i} \hat{f}_{i}, \quad t \in(\mathbb{R} \cup\{ \pm \infty\}) \backslash \widehat{R}_{q}^{-1}(0),
$$

where

$$
\widehat{R}_{q}(t)=t^{k}+\sum_{i=1}^{k-2} a_{i}(q) t^{i}+a_{1}(q), \quad a_{1}(q) \neq 0 .
$$

A possible construction of the above objects is described in Section 5.

Example 1 If the assumptions of Theorem 3 are satisfied for $D \in \mathcal{A}_{0}(8,6)$, then there exist a canonical function germ $a$ and germs of vector fields $\hat{f}_{0}, \ldots, \hat{f}_{3}$ such that the fibers $S_{q}^{D}$ have parametrizations:

$$
t \mapsto \hat{f}_{0}+\frac{t}{t^{3}+a(q)(t+1)}\left(\hat{f}_{1}+t \hat{f}_{2}+t^{2} \hat{f}_{3}\right)
$$

Theorem 4 If a distribution $D \in \mathcal{A}_{0}(6,4)$ satisfies non-degeneracy Conditions (G1), (G2) together with Conditions (G4) and (G5), then there is a canonical way of defining the following objects: vector fields $\hat{f}_{0}, \hat{f}_{2}$; a coefficient $\alpha \in\{0,1\}$; and a vector field $\hat{f}_{1}$ that is determined up to multiplication by \pm 1 (independently of $\alpha$ ), such that:

$$
C^{D}=\hat{f}_{0}+\operatorname{span}\left\{\hat{f}_{1}, \hat{f}_{2}\right\}
$$

and the fibers $S_{q}^{D}$ have parametrizations:

$$
t \mapsto \hat{f_{0}}+\frac{t}{t^{2}+\alpha}\left(\hat{f}_{1}+t \hat{f}_{2}\right), \quad t \in \mathbb{R} \cup\{ \pm \infty\}
$$

The above theorem is illustrated in Figs. 1 and 2.

The paper is organized as follows. In the next section, we study relations between affine distributions, their cogenerators, and singular velocities. In Section 3, we prove Theorem 2. An example of a distribution satisfying assumptions of this theorem is presented in Section 4. Finally, in Section 5, we construct some canonical objects related to an affine distribution. In particular, Theorems 3 and 4 are proved there.

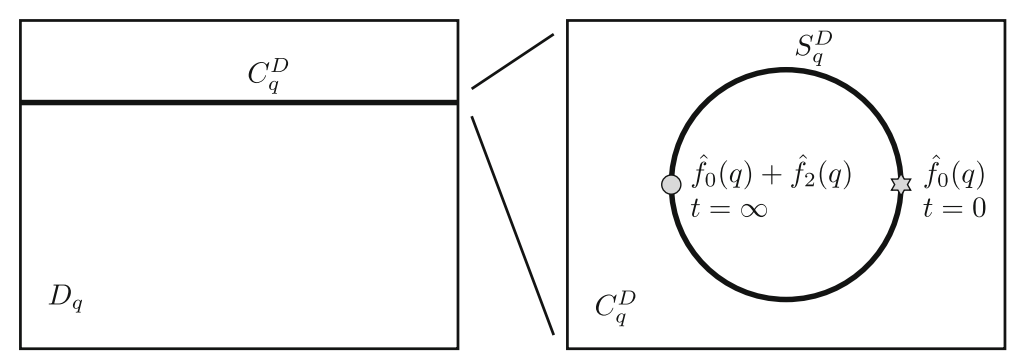

Fig. 1 For $D \in \mathcal{A}_{0}(6,4)$ and $\alpha=1, S^{D}$ is a bundle of circles 


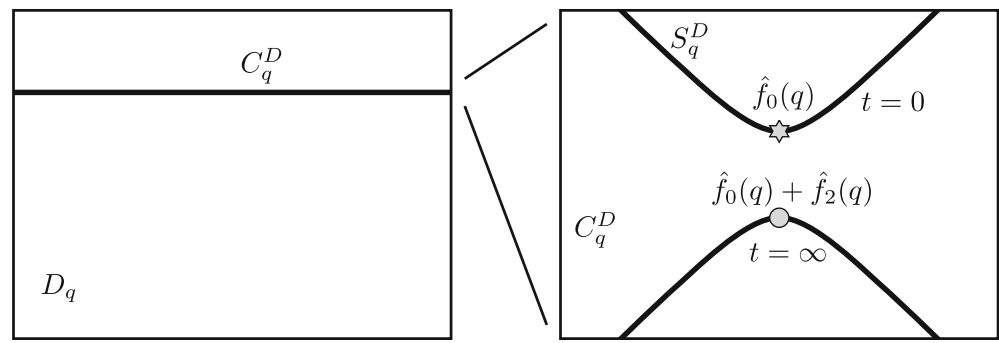

Fig. 2 For $D \in \mathcal{A}_{0}(6,4)$ and $\alpha=-1, S^{D}$ is a bundle of hyperbolas

\section{Singular Velocities}

A singular curve of a distribution $D \in \mathcal{A}(n, l)$ is an absolutely continuous curve

$$
[0, T]=I \ni t \mapsto q_{t} \in M,
$$

that is $D$-horizontal, i.e., almost everywhere tangential to $D$, and that is a singular point of the endpoint mapping that maps horizontal curves (with fixed $T$ ) to their endpoints. We refer the reader to [4] and [17, Appendix D] for details on the differential structure of the space of horizontal curves. The initial velocity of a singular curve will be called a singular velocity. The set of such velocities over a point $q \in M$ is denoted by $S_{q}^{D}$.

The main tool of the analysis of singular velocities is the following theorem due to Hsu [11] (a version of this theorem that is particularly adapted for affine distributions may be found in [7]). This theorem constitutes a precise formulation of the already mentioned relation: a singular curve is a projection of a singular extremal, and it is based on the classical Pontryagin Maximum Principle [18].

Theorem 5 A vector $v \in T_{q_{0}} M$ is a singular velocity for a distribution $D \in \mathcal{A}(M, l)$ if and only if there exists a D-horizontal curve

$$
I \ni t \mapsto q_{t} \in M
$$

with a lift to the cotangent bundle

$$
I \ni t \mapsto \lambda_{t}=\left(q_{t}, p_{t}\right) \in T^{*} M, \quad p_{t} \in T_{q_{t}}^{*} M
$$

satisfying the following conditions:

(i) $\lambda$ is an integral curve of a Hamiltonian system with Hamiltonian of the form

$$
H_{u}(q, p)=h_{0}(q, p)+\sum_{i=1}^{l} u_{i}(q) h_{i}(q, p), \quad p \in T_{q}^{*} M,
$$

where, for a fixed system $\left(f_{0}, f_{1}, \ldots, f_{l}\right)$ of generators of $D$,

$$
\left.h_{i}(q, p)=f_{i}(q)\right\lrcorner p, \quad p \in T_{q}^{*} M,
$$

and

$$
v=\dot{q}_{0}=f_{0}\left(q_{0}\right)+u_{1}\left(q_{0}\right) f_{1}\left(q_{0}\right)+\cdots+u_{l}\left(q_{0}\right) f_{l}\left(q_{0}\right) .
$$

(ii) $p_{t}$ does not vanish, i.e.,

$$
p_{t} \neq 0, \quad \text { for } t \in I \text {. }
$$


(iii) There exists a constant $c \in\{0,1\}$ such that

$$
h_{0}\left(\lambda_{t}\right)=c \quad \text { and } \quad h_{j}\left(\lambda_{t}\right)=0, \quad \forall t \in I, \quad j=1, \ldots, l .
$$

We say that a 1-form $\omega$ polarizes a distribution $D$, if it satisfies the following condition:

$$
\forall X \in \Gamma(D): X\lrcorner \omega=1,
$$

where $\Gamma(D)$ denotes a section of $D$ (i.e., a vector field). Similarly, we will write that a 1-form $\omega$ annihilates $D$ if

$$
\forall X \in \Gamma(D): X\lrcorner \omega=0 .
$$

For brevity's sake, we will write $\omega \in D^{=}$to state that a 1-form $\omega$ either annihilates or polarizes distribution $D$.

Corollary 6 If $D \in \mathcal{A}_{q}(n, l)$ and $v \in D_{q}$ is a singular velocity of $D$, then there exists a non-vanishing 1-form $\omega \in D^{=}$such that

$$
\left.\left.\bar{D}_{q}\right\lrcorner v\right\lrcorner d \omega(q)=0 .
$$

The above corollary will be proved with a help of the following lemma, which is a direct consequence of a well-known formula:

$$
\left.\left.\left.\left.\left.X_{1}\right\lrcorner X_{2}\right\lrcorner d \omega=\left[X_{1}, X_{2}\right]\right\lrcorner \omega+X_{2}\left(X_{1}\right\lrcorner \omega\right)-X_{1}\left(X_{2}\right\lrcorner \omega\right) .
$$

Lemma 7 Let $\omega$ be a differential 1-form and $X_{1}$ and $X_{2}$ be the two vector fields such that the inner products $\left.X_{1}\right\lrcorner \omega$ and $\left.X_{2}\right\lrcorner \omega$ are constant functions. Then,

$$
\left.\left.\left.X_{1}\right\lrcorner X_{2}\right\lrcorner d \omega=\left[X_{1}, X_{2}\right]\right\lrcorner \omega .
$$

Proof of Corollary 6 Let $\lambda_{t}=\left(q_{t}, p_{t}\right)$ be as in Theorem 5 and let $\omega \in D^{=}$be a form that coincides with $p_{0}$ at $q_{0}$. Differentiating (5) along $\lambda_{t}$, we get

$$
\left\{H_{u}, h_{j}\right\}\left(\lambda_{0}\right)=0, \quad j=0,1, \ldots, l,
$$

i.e., setting $u_{0} \equiv 1$,

$$
\left.\left.0=\sum_{i=0}^{l} u_{i}(q)\left[f_{i}, f_{j}\right](q)\right\lrcorner p_{0}=\sum_{i=0}^{l} u_{i}(q)\left[f_{i}, f_{j}\right](q)\right\lrcorner \omega(q),
$$

The proof is completed by invoking Lemma 7.

For $D \in \mathcal{A}_{q_{0}}(2 k+2,2 k)$, we define set $D_{\text {reg }}^{=}$as

$$
D_{\text {reg }}^{=}=\left\{\omega \in D^{=}\left|d \omega^{l}\right|_{\underline{D}} \neq 0\right\} .
$$

Proposition 8 If $D \in \mathcal{A}_{q_{0}}(2 k+2,2 k)$ and $\omega \in D_{\text {reg }}^{=}$, then the unique vector $v \in D_{q_{0}}$ satisfying (7), i.e.,

$$
\left.\left.\bar{D}_{q_{0}}\right\lrcorner v\right\lrcorner d \omega\left(q_{0}\right)=0,
$$

is a singular velocity of distribution $D$.

If the assumptions of the above proposition are satisfied, then by extending (8) to nearby points we obtain a germ of vector field $X_{\omega}$ that consists of singular velocities of distribution $D$ :

$$
\left.\left.\bar{D}_{q}\right\lrcorner X_{\omega}(q)\right\lrcorner d \omega(q)=0, \quad X_{\omega}(q) \in D_{q} .
$$


Proof of Proposition 8 The existence and uniqueness of $v$ come from the facts that $\bar{D}_{q}$ is odd-dimensional and $\left.d \omega^{k}\left(q_{0}\right)\right|_{\underline{D}} \neq 0$. It remains to prove that $v \in S_{q_{0}}^{D}$. Let

$$
v=f_{0}\left(q_{0}\right)+\sum_{i=1}^{2 k} v_{i} f_{i}\left(q_{0}\right)
$$

Using Lemma 7, we get

$$
\left.\left(\left[f_{0}, f_{j}\right]\left(q_{0}\right)+\sum_{i=1}^{2 k} v_{i}\left[f_{i}, f_{j}\right]\left(q_{0}\right)\right)\right\lrcorner \omega\left(q_{0}\right)=0, \quad j=1, \ldots, 2 k .
$$

Consider the equations

$$
\left.\left(\left[f_{0}, f_{j}\right](q)+\sum_{i=1}^{2 k} U_{i}\left[f_{i}, f_{j}\right](q)\right)\right\lrcorner p=0, \quad p \in T_{q}^{*} M, \quad j=1, \ldots, 2 k,
$$

where, for brevity, we write $M$ for $\mathbb{R}^{2 k+2}$. By the implicit function theorem, there exist unique functions $U_{1}, \ldots, U_{2 k}$ such that

$$
\left(U_{1}\left(q_{0}, p_{0}\right), \ldots, U_{2 k}\left(q_{0}, p_{0}\right)\right)=\left(v_{1}, \ldots, v_{2 k}\right)
$$

and (9) holds in a neighborhood of

$$
\left(q_{0}, p_{0}\right)=\left(q_{0}, \omega\left(q_{0}\right)\right) \in T^{*} M,
$$

provided that skew-symmetric matrix

$$
\left.\left(\left[f_{i}, f_{j}\right]\left(q_{0}\right)\right\lrcorner p_{0}\right)_{i, j=1, \ldots, 2 k}
$$

is of full rank, which is implied by the fact $\omega \in D_{\text {reg }}^{=}$. Let $\lambda_{t}=\left(q_{t}, p_{t}\right)$ be the integral curve of the Hamiltonian system with Hamiltonian

$$
\widehat{H}(q, p)=h_{0}(q, p)+\sum_{i=1}^{2 k} U_{i}(q, p) f_{i}(q), \quad p \in T_{q}^{*} M .
$$

This curve is also an integral curve of the Hamiltonian system with Hamiltonian

$$
H(q, p)=h_{0}(q, p)+\sum_{i=1}^{2 k} u_{i}(q) f_{i}(q), \quad p \in T_{q}^{*} M,
$$

where $u_{i}, i=1, \ldots, 2 k$ represent any functions satisfying equations

$$
u_{i}\left(q_{t}\right)=U_{i}\left(\lambda_{t}\right), \quad i=1, \ldots, 2 k .
$$

By Theorem 5, $v=\dot{q}_{0} \in S_{q_{0}}^{D}$.

\section{Proof of the Local Determinacy Theorem}

Propositions 1 and 8 prove the first part of Theorem 2, i.e., that Conditions (G2) and (G2) imply

$$
C^{D}=L \cap D=\operatorname{aff} \text {-span } S^{D} \in \mathcal{A}_{q}(2 k+2, k)
$$

The following two lemmas complete the proof of Theorem 2.

Lemma 9 Let $D \in \mathcal{A}_{q}(2 k+2,2 k)$ satisfy Conditions (G1) and (G2) and let $C=$ aff-span $S^{D}$. Then $[C, C] \subset \underline{D}$. 
Lemma 10 If $D \in \mathcal{A}_{q_{0}}(2 k+2,2 k)$ satisfies Conditions (G1)-(G3), then $C^{D}=$ aff-span $S^{D}$ is the unique rank $k$ distribution satisfying equality

$$
C+[C, C]=D \text {. }
$$

Proof of Lemma 9 Let $p_{0}, p_{1}$ constitute an affine basis of $D_{q}^{\top}$. By Propositions 1 and 8 , there exist $k+1$ numbers $t_{0}, \ldots, t_{k}$ and $k+1$ independent vectors $v_{0}, \ldots, v_{k} \in D_{q}$ such that each $v_{i}$ belongs to the kernel of

$$
\overline{\mathrm{A}}_{q}^{*}(\underbrace{t_{i} p_{1}+\left(1-t_{i}\right) p_{0}}_{v_{i}}) .
$$

Let $X_{i}$ be a vector field tangent to $C$ and coinciding with $v_{i}$ at $q$. By definition of $\overline{\mathrm{A}}_{q}^{*}$, we have

$$
\left[X_{i}, X_{j}\right](q) \in\left(\operatorname{ker} v_{i}\right) \cap\left(\operatorname{ker} v_{j}\right)=\underline{D}_{q}
$$

Proof of Lemma 10 Conditions (G1) and (G2) imply (see the discussion following Proposition 1)

$$
C^{D}=L \cap D=\operatorname{aff-span} S^{D} \in \mathcal{A}_{q}(2 k+2, k) .
$$

Thus, it is enough to show that $S_{q}^{D} \subset C$. Let $\left(f_{0}, f_{1}, \ldots, f_{2 k}\right)$ be a system of generators of $D$ such that

$$
C=f_{0}+\operatorname{span}\left\{f_{1}, \ldots, f_{k}\right\}
$$

Equation (10) implies that $[C, C] \subset \underline{D}$ and thus

$$
\left.\left[f_{i}, f_{j}\right]\right\lrcorner \omega=0, \quad \omega \in D^{=}, \quad i, j=0,1, \ldots, k .
$$

According to Corollary 6 and Proposition 1, if $v=f_{0}\left(q_{0}\right)+\sum_{i=1}^{2 k} v_{i} f_{i}\left(q_{0}\right) \in S_{q}^{D}$, then there exists $\omega \in D_{\text {reg }}^{=}$such that the following equations hold:

$$
\left.\left.\left(\sum_{i=1}^{2 k} v_{i}\left[f_{j}, f_{i}\right]\left(q_{0}\right)\right)\right\lrcorner \omega\left(q_{0}\right)=\left(\left[f_{0}, f_{j}\right]\right\lrcorner \omega\right)\left(q_{0}\right), \quad j=1, \ldots, 2 k .
$$

Therefore, vector $V=\left(v_{1}, \ldots, v_{2 k}\right)^{T}$ must satisfy the equation

$$
A\left(q_{0}\right) V=B\left(q_{0}\right)
$$

where $\left.\left.A=\left(\left[f_{i}, f_{j}\right]\right\lrcorner \omega\right)_{i=1, \ldots, 2 k}^{j=1, \ldots, 2 k}, B=\left(\left[f_{0}, f_{j}\right]\right\lrcorner \omega\right)^{j=1, \ldots, 2 k}$. We have

$$
\left.A\left(q_{0}\right)=\left(\begin{array}{cc}
0 & R\left(q_{0}\right) \\
-R^{T}\left(q_{0}\right) & *
\end{array}\right), \quad B\left(q_{0}\right)=\left(\begin{array}{c}
0 \\
*
\end{array}\right), \quad R=\left(\left[f_{i}, f_{j}\right]\right\lrcorner \omega\right)_{i=1, \ldots, k}^{j=k+1, \ldots, 2 k} .
$$

Due to the regularity of $\omega, \operatorname{det} A\left(q_{0}\right) \neq 0$ and thus $v_{i}=0$ for $i=k+1, \ldots, 2 k$, which means that $v \in C_{q_{0}}$. 


\section{An Example}

Let $n=2 k+2$ and

$$
\begin{aligned}
\omega_{0}= & d x_{1}+d x_{2}+d x_{4}+\cdots+d x_{n}+x_{2} d x_{3}+x_{4} d x_{5}+\cdots+x_{n-2} d x_{n-1} \\
\omega_{1}= & d x_{2}+d x_{4}+\cdots+d x_{n}+x_{3} d x_{4}+x_{5} d x_{6}+\cdots+x_{n-1} d x_{n}+ \\
& +x_{n}\left(x_{2} d x_{4}+x_{4} d x_{6}+\cdots+x_{n-2} d x_{n}\right) .
\end{aligned}
$$

In this section, we show that the above pair $\mathcal{K}=\left(\omega_{0}, \omega_{1}\right)$ of 1 -forms constitutes a system of cogenerators of an affine distribution $D \in \mathcal{A}_{0}(2 k+2,2 k)$ satisfying the assumptions (Conditions (G1)-(G3)) of Theorem 2. The presentation is divided into three subsections related to the Conditions (G1), (G2), and (G3), respectively.

In what follows, we will use the following technical lemma. We leave its proof to the reader (one may also find it in [19]).

Lemma 11 Consider the following differential forms defined on $\mathbb{R}^{n}=\mathbb{R}^{2 k+2}$ :

$$
\begin{aligned}
\alpha & =d x_{2} \wedge d x_{3}+d x_{4} \wedge d x_{5}+\cdots+d x_{n-2} \wedge d x_{n-1}, \\
\beta & =d x_{3} \wedge d x_{4}+d x_{5} \wedge d x_{6}+\cdots+d x_{n-1} \wedge d x_{n}, \\
\gamma & =d x_{2} \wedge d x_{4}+d x_{4} \wedge d x_{6}+\cdots+d x_{n-2} \wedge d x_{n}, \\
\delta & =d x_{4} \wedge d x_{n}+d x_{6} \wedge d x_{n}+\cdots+d x_{n-2} \wedge d x_{n} .
\end{aligned}
$$

Then, for each $0 \leq s \leq k$ and $0 \leq i \leq k-1$,

(b) $\alpha^{i} \wedge \gamma \wedge \beta^{k-i-1}=i !(k-i-1) ! \Omega / d x_{1} / d x_{2 i+3}$,

(c) $\alpha^{i} \wedge \delta \wedge \beta^{k-i-1}=i !(k-i-1) ! \sum_{j=1}^{i} \Omega / d x_{1} / d x_{n-2(i-j)-1}$,

where $\Omega / d x_{1} / d x_{a}$ denotes the product $d x_{2} \wedge d x_{3} \wedge \cdots \wedge d x_{n}$ with factor $d x_{a}$ omitted.

\subsection{Condition (G1)}

One-forms $\omega_{0}$ and $\omega_{1}$ are linearly independent at the origin (so they cogenerate a corank 2 distribution), and we have

$$
\begin{aligned}
& \left.\omega_{0} \wedge \omega_{1}=d x_{1} \wedge \omega_{1}+\text { (terms without } d x_{1} \text { factor }\right)= \\
& =d x_{1} \wedge d x_{2}+d x_{1} \wedge d x_{4}+\cdots+d x_{1} \wedge d x_{n}+ \\
& +x_{3} d x_{1} \wedge d x_{4}+x_{5} d x_{1} \wedge d x_{6}+\cdots+x_{n-1} d x_{1} \wedge d x_{n}+ \\
& +x_{n}\left(x_{2} d x_{1} \wedge d x_{4}+x_{4} d x_{1} \wedge d x_{6}+\cdots+x_{n-2} d x_{1} \wedge d x_{n}\right)+ \\
& + \text { (terms without } d x_{1} \text { factor). } \\
& \omega_{0}-\omega_{1}=d x_{1}+x_{2} d x_{3}+x_{4} d x_{5}+\cdots+x_{n-2} d x_{n-1}+ \\
& -\left(x_{3}+x_{2} x_{n}\right) d x_{4}-\left(x_{5}+x_{4} x_{n}\right) d x_{6}-\cdots-\left(x_{n-1}+x_{n-2} x_{n}\right) d x_{n}, \\
& d \omega_{0}=d x_{2} \wedge d x_{3}+d x_{4} \wedge d x_{5}+\cdots+d x_{n-2} \wedge d x_{n-1} \text {, } \\
& d \omega_{1}=\underbrace{d x_{3} \wedge d x_{4}+d x_{5} \wedge d x_{6}+\cdots+d x_{n-1} \wedge d x_{n}}_{\bullet}+
\end{aligned}
$$




$$
\begin{aligned}
& +\underbrace{x_{n}\left(d x_{2} \wedge d x_{4}+d x_{4} \wedge d x_{6}+\cdots+d x_{n-2} \wedge d x_{n}\right)}+ \\
& +\underbrace{d x_{n} \wedge\left(x_{2} d x_{4}+x_{4} d x_{6}+\cdots+x_{n-2} d x_{n}\right)} .
\end{aligned}
$$

By Lemma 11(a):

$$
d \omega_{0}^{k}=k ! d x_{2} \wedge d x_{3} \wedge \cdots \wedge d x_{n-1} .
$$

For each $i=0,1, \ldots, k-1$, the form

$$
\begin{array}{r}
d \omega_{0}^{i} \wedge d \omega_{1}^{k-i}=d \omega_{0}^{i} \wedge(\bullet)^{k-i}+d \omega_{0}^{i} \wedge(k-i)(\bullet+\mathbf{\Delta}) \wedge(\bullet)^{k-i-1}= \\
=d \omega_{0}^{i} \wedge(\bullet)^{k-i}+(k-i) d \omega_{0}^{i} \wedge(\bullet) \wedge(\bullet)^{k-i-1}+(k-i) d \omega_{0}^{i} \wedge(\boldsymbol{\Delta}) \wedge(\bullet)^{k-i-1} .
\end{array}
$$

can be written as a linear combination of $(n-2)$-forms:

$$
\Omega / d x_{1} / d x_{a}, \quad 1<a \leq n .
$$

If $a$ is even, then $\Omega / d x_{1} / d x_{a}$ can result only from $d \omega_{0}^{i} \wedge(\bullet)^{k-i}$. If $a$ is odd, then $\Omega / d x_{1} / d x_{a}$ can result only from those summands of $d \omega_{0}^{i} \wedge d \omega_{1}^{k-i}$ in which there is exactly one factor from the part of $d \omega_{1}$ denoted by $\boldsymbol{\nabla}$. In other words, for odd $a$, the form $\Omega / d x_{1} / d x_{a}$ can result only from products

$$
d \omega_{0}^{i} \wedge(\diamond+\mathbf{\Delta}) \wedge(\bullet)^{k-i-1}, \quad i=0,1, \ldots, k-1 .
$$

Due to Lemma 11, we thus have, for $i=0,1, \ldots, k-1$,

$d \omega_{0}^{i} \wedge d \omega_{1}^{k-i}=i !(k-i) !\left(\Omega / d x_{1} / d x_{2 i+2}+x_{n} \Omega / d x_{1} / d x_{2 i+3}-\sum_{j=1}^{i} x_{2 j} \Omega / d x_{1} / d x_{n-2(i-j)-1}\right)$.

Using equalities equations (12), (13), and (15), we get that the forms

$$
\omega_{0} \wedge \omega_{1} \wedge d \omega_{0}^{i} \wedge d \omega_{1}^{k-i}, \quad i=0,1, \ldots, k
$$

equal:

$k ! \Omega$,

$$
\text { for } i=0 \text {, }
$$$$
i !(k-i) !\left(1+x_{2 i+1}+x_{2 i} x_{n}\right) \Omega, \quad \text { for } i=1, \ldots, k-1,
$$

$k !\left(1+x_{n-1}+x_{n-2} x_{n}\right) \Omega$,

for $i=k$,

where $\Omega=d x_{1} \wedge \cdots \wedge d x_{n}$. Condition (G1) is satisfied because all the coefficients of the polynomial mapping (3) are non-zero.

\subsection{Condition (G2)}

As in the previous subsection, we may show that the forms

$$
\left(\omega_{0}-\omega_{1}\right) \wedge d \omega_{0}^{i} \wedge d \omega_{1}^{k-i} /(i !(k-i) !), \quad i=0,1, \ldots, k
$$

equal:

$$
\begin{array}{cl}
\Omega / d x_{2 i+2}+x_{n} \Omega / d x_{2 i+3}-x_{2 i+2} x_{n} \Omega / d x_{1}, & \text { for } i=0, \\
\Omega / d x_{n}-\left(x_{n-1}+x_{n-2} x_{n}\right) \Omega / d x_{1}, & \text { for } i=k,
\end{array}
$$


and for $1 \leq i \leq k-1$ they equal

$$
\begin{gathered}
\Omega / d x_{2 i+2}+x_{n} \Omega / d x_{2 i+3}-\sum_{j=1}^{i} x_{2 j} \Omega / d x_{n-2(i-j)-1} \\
-\left(x_{2 i+1}+x_{2 i} x_{n}+x_{2 i+2} x_{n}-\sum_{j=1}^{i} x_{2 j} x_{n-2(i-j)-2}\right) \Omega / d x_{1},
\end{gathered}
$$

where $\Omega / d x_{i}=d x_{1} \wedge \cdots \wedge d x_{i-1} \wedge d x_{i+1} \wedge \cdots \wedge d x_{n}$. Clearly, the above forms are linearly independent at the origin and Condition (G2) is satisfied due to the following lemma.

Lemma 12 Let $\omega_{0}$ and $\omega_{1}$ be affine cogenerators of a distribution $D \in \mathcal{A}_{q}(2 k+2,2 k)$. Condition $(\mathrm{G} 2)$ is equivalent to the linear independence (at q) of the following forms:

$$
\left(\omega_{1}-\omega_{0}\right) \wedge d \omega_{0}^{i} \wedge d \omega_{1}^{k-i}, \quad i=0, \ldots, k
$$

Proof $D_{q}^{\top}$ may be parametrized as:

$$
\mathbb{R} \ni t \mapsto p_{t}=\omega_{t}(q)=t \omega_{1}(q)+(1-t) \omega_{0}(q) .
$$

The kernel $V_{p_{t}}$ of $\underline{\mathrm{A}}_{q}^{*}\left(p_{t}\right)$ is the kernel of the 2-form $t d \omega_{1}+(1-t) d \omega_{0}$ evaluated at $q$ and restricted to $\bar{D}_{q} \wedge \bar{D}_{q}$. Thus, the kernel $V_{p_{t}}$ is exactly the kernel of the following ( $2 k+$ 1)-form evaluated at $q$ :

$$
\left(\omega_{1}-\omega_{0}\right) \wedge\left(t d \omega_{1}+(1-t) d \omega_{0}\right)^{k}=\left(\omega_{1}-\omega_{0}\right) \wedge \sum_{i=0}^{k}\left(\begin{array}{l}
k \\
i
\end{array}\right)(1-t)^{i} t^{k-i} d \omega_{0}^{i} \wedge d \omega_{1}^{k-i} .
$$

Let $\Omega$ be a local volume form and let vector fields $Z_{i}, i=0, \ldots, k$ be defined as follows:

$$
\left.Z_{i}\right\lrcorner \Omega=\left(\omega_{1}-\omega_{0}\right) \wedge d \omega_{0}^{i} \wedge d \omega_{1}^{k-i} .
$$

Then, the kernel $V_{p_{t}}$ is linearly spanned by

$$
\left(\begin{array}{l}
k \\
i
\end{array}\right)(1-t)^{i} t^{k-i} Z_{i}(q)
$$

and the assertion of the lemma is proved by invoking Proposition 1.

\subsection{Condition $(\mathrm{G} 3)$}

Computations performed in the two previous subsections show that vector fields $Y_{i}, i=$ $0, \ldots, k$, defined by the relations

$$
\left.Y_{i}\right\lrcorner\left(\omega_{0} \wedge \omega_{1} \wedge d \omega_{0}^{i} \wedge d \omega_{1}^{k-i}\right)=\left(\omega_{1}-\omega_{0}\right) \wedge d \omega_{0}^{i} \wedge d \omega_{1}^{k-i}, \quad i=0, \ldots, k
$$

are linearly independent and span distribution $L$ introduced in Proposition 1 . These vector fields also are sections of the affine distribution $D$, for if we multiply (from the left) both sides of Eq. (24) by $\omega_{r}, r=0,1$, we get

$$
\left.\left(Y_{i}\right\lrcorner \omega_{r}\right) \wedge\left(\omega_{0} \wedge \omega_{1} \wedge d \omega_{0}^{i} \wedge d \omega_{1}^{k-i}\right)=\omega_{0} \wedge \omega_{1} \wedge d \omega_{0}^{i} \wedge d \omega_{1}^{k-i}
$$


and thus $\left.Y_{i}\right\lrcorner \omega_{r}=1$. Hence, vector fields $Y_{i}, i=0, \ldots, k$, affinely span distribution $C^{D}$. By Eqs. 17-22, we have:

$$
\begin{aligned}
& Y_{0}=-\frac{\partial}{\partial x_{2}}+x_{n} \frac{\partial}{\partial x_{3}}-x_{2} x_{n} \frac{\partial}{\partial x_{1}}, \\
& Y_{k}=\frac{-1}{1+x_{n-1}+x_{n-2} x_{n}}\left(\frac{\partial}{\partial x_{n}}+\left(x_{n-1}+x_{n-2} x_{n}\right) \frac{\partial}{\partial x_{1}}\right),
\end{aligned}
$$

and, for $i=1,2, \ldots, k-1$,

$$
\begin{array}{r}
Y_{i}=\frac{-1}{1+x_{2 i+1}+x_{2 i} x_{n}}\left(\frac{\partial}{\partial x_{2 i+2}}-x_{n} \frac{\partial}{\partial x_{2 i+3}}+\sum_{j=1}^{i} x_{2 j} \frac{\partial}{\partial x_{n-2(i-j)-1}}+\right. \\
+\left(x_{2 i+1}+x_{2 i} x_{n}+x_{2 i+2} x_{n}+\sum_{j=1}^{i} x_{2 j} x_{n-2(i-j)-2) \frac{\partial}{\partial x_{1}}}\right) .
\end{array}
$$

In particular,

$$
\begin{aligned}
Y_{i}(0) & =-\frac{\partial}{\partial x_{2 i+1}}, & i & =0,1, \ldots, k, \\
{\left[Y_{k}, Y_{i}\right](0) } & =-\frac{\partial}{\partial x_{2 i+3}}, & i & =0,1, \ldots, k-1 .
\end{aligned}
$$

and therefore

$$
\operatorname{dim}\left(C_{0}^{D}+\left[C^{D}, C^{D}\right]_{0}\right) \geq 2 k
$$

In fact, the above inequality must be strict because

$$
C_{0}^{D}+\left[C^{D}, C^{D}\right]_{0} \subset D_{0}
$$

\section{Normalization}

In this section, we provide results that form Theorems 3 and 4.

Let $D \in \mathcal{A}_{q}(2 k+2,2 k)$ be a distribution satisfying Conditions (G1) and (G2) and let $\mathcal{K}=$ $\left(\omega_{0}, \omega_{1}\right)$ be its cogenerators. The Pfaffian of the pencil $\underline{A}^{*}$ can be viewed as a polynomial in one variable:

$$
t \mapsto \omega=t \omega_{1}+(1-t) \omega_{0} \mapsto R_{\omega},
$$

where $R_{\omega}$ is the determinant of the matrix $R$ appearing in Eq. (11). By $R_{\mathcal{K}}(t)$, we will denote this Pfaffian normalized to a monic polynomial (i.e., with the leading coefficient equal to 1). This normalization can be always performed by an appropriate choice of $\underline{D}$ generators. The polynomial $R_{\mathcal{K}}$ is uniquely determined by the distribution $D$ up to an affine change in variable $t$ (which comes from the same freedom in parameterizing the affine line $D^{\top}$ ). We will refer to this polynomial as $R_{D}$ whenever the choice of cogenerators is irrelevant. In particular, for a distribution $D \in \mathcal{A}_{q}(2 k+2,2 k)$, the following conditions make sense independently of the freedom above:

$R_{D}$ is of degree $k$,

the arithmetic mean of the roots of $R_{D}$ is not a root of $R_{D}$,

the mean of the roots of $R_{D}$ is not a root of $R_{D}$ 's first derivative.

Remark 13 One can show that Condition $(\mathrm{G} 4)$ is equivalent to the fact that the characteristic line field (see [15]) of the corank 1 distribution $\bar{D}$ is not parallel to the corank 2 distribution $\underline{D}$. 
Remark 14 In what follows, we use Conditions (G4)-(G6) to ensure the existence of canonical cogenerators of $D$ and canonical generators of $C^{D}$. As the anonymous reviewer notice, if we were satisfied with cogenerators and generators defined canonically up to a specific action of $\mathbb{Z}_{2}$, we could drop Condition (G6) and replace Conditions (G4) and (G5) with their weaken versions:

$R_{D}$ is of constant degree $d>1$,

the arithmetic mean of the roots of $R_{D}$ is not a root of $R_{D}$

or it is a root of constant multiplicity $m<d$,

Before going into further analysis, let us present an example of distributions satisfying and not satisfying Conditions (G4)-(G6).

Example 2 Let $D \in \mathcal{A}_{0}(2 k+2,2 k), n=2 k+2 \geq 4$, be a distribution with cogenerators $\mathcal{K}=\left(\omega_{0}, \omega_{1}\right)$ defined in Section 4. We have (see Section 4):

$$
\begin{aligned}
d \omega_{0}(0) & =d x_{2} \wedge d x_{3}+d x_{4} \wedge d x_{5}+\cdots+d x_{n-2} \wedge d x_{n-1}, \\
d \omega_{1}(0) & =d x_{3} \wedge d x_{4}+d x_{5} \wedge d x_{6}+\cdots+d x_{n-1} \wedge d x_{n}, \\
C_{0}^{D} & =\text { aff-span }\left\{-\frac{\partial}{\partial x_{2}},-\frac{\partial}{\partial x_{4}}, \ldots,-\frac{\partial}{\partial x_{n}}\right\}, \\
D_{0} & =C_{0}^{D}+\operatorname{span}\left\{\frac{\partial}{\partial x_{3}}, \frac{\partial}{\partial x_{5}}, \ldots, \frac{\partial}{\partial x_{n-1}}\right\} .
\end{aligned}
$$

The matrices $R_{\omega_{i}}, i=0,1$ (constructed at $q=0$ ) can be computed using the formula:

$$
\left.\left.R_{\omega_{i}}=\left(f_{j}\right\lrcorner f_{r}\right\lrcorner d \omega_{i}\right)_{r=1,2, \ldots, k}^{j=k+1, k+2, \ldots, k+k},
$$

where $f_{i}, i=0,1, \ldots, 2 k$ are as in the proof of Lemma 10. We may take:

$$
\begin{aligned}
& f_{1}(0)=\frac{\partial}{\partial x_{n}}-\frac{\partial}{\partial x_{2}}, f_{2}(0)=\frac{\partial}{\partial x_{n}}-\frac{\partial}{\partial x_{4}}, \ldots, f_{k}(0)=\frac{\partial}{\partial x_{n}}-\frac{\partial}{\partial x_{2 k}}, \\
& f_{k+1}(0)=-\frac{\partial}{\partial x_{3}}, \quad f_{k+2}(0)=-\frac{\partial}{\partial x_{5}}, \quad \ldots, f_{2 k}(0)=-\frac{\partial}{\partial x_{n-1}} .
\end{aligned}
$$

Thus, $R_{\omega_{0}}$ becomes the $k$ by $k$ identity matrix and matrices $R_{\omega_{1}}$ and $t R_{\omega_{1}}+(1-t) R_{\omega_{0}}$ are, respectively,

$$
\left(\begin{array}{llllll}
0 & 0 & 0 & \ldots & 0 & 1 \\
-1 & 0 & 0 & \ldots & 0 & 1 \\
0 & -1 & 0 & \ldots & 0 & 1 \\
\vdots & \ddots & \ddots & \ddots & \vdots & \vdots \\
0 & \ldots & 0 & -1 & 0 & 1 \\
0 & \ldots & 0 & 0 & -1 & 1
\end{array}\right), \quad\left(\begin{array}{llllll}
1-t & 0 & 0 & \ldots & 0 & t \\
-t & 1-t & 0 & \ldots & 0 & t \\
0 & -t & 1-t & \ldots & 0 & t \\
\vdots & \ddots & \ddots & \ddots & \vdots & \vdots \\
0 & \ldots & 0 & -t & 1-t & t \\
0 & \ldots & 0 & 0 & -t & 1
\end{array}\right) .
$$

The determinant of the latter matrix can be computed using Laplace expansion with respect to the last column. In this way, we obtain, up to normalization to a monic polynomial:

$$
\begin{array}{r}
R_{\mathcal{K}}(t)=\operatorname{det}\left(t R_{\omega_{1}}+(1-t) R_{\omega_{0}}\right)=(1-t)^{k-1}+\sum_{i=1}^{k-1}(1-t)^{i-1} t^{k-i+1}= \\
=(1-t)^{k-1}(1-t+t)+\sum_{i=0}^{k-2}(1-t)^{i} t^{k-i}=\sum_{i=0}^{k}(1-t)^{i} t^{k-i}=\frac{(1-t)^{k+1}-t^{k+1}}{1-2 t} .
\end{array}
$$


In particular, $R_{\mathcal{K}}(t)$ is of degree $k$ (Condition (G4) is satisfied) if $k$ is even, and it is of degree $k-1$ ( (G4) fails) if $k$ is odd. Moreover, $R_{\mathcal{K}}$ is invariant with respect to the change of variable

$$
t \mapsto 1-t,
$$

and thus the average of all $R_{\mathcal{K}}$ roots is $1 / 2$. Since

$$
R_{\mathcal{K}}\left(\frac{1}{2}\right)=\frac{k+1}{2^{k}} \neq 0=R_{\mathcal{K}}^{\prime}\left(\frac{1}{2}\right),
$$

Condition (G5) is satisfied whereas Condition (G6) is not.

We can fix the "origin" (one-form $\omega_{0}$ ) on $D^{\top}$ by making the sum of $R_{\mathcal{K}}$ roots equal to zero or equivalently by making $a_{k-1}=0$. Then, we may fix the "scale" (one-form $\omega_{1}-\omega_{0}$ ) on $D^{\top}$ by making $a_{1}=a_{0}$ for $k>2$ (this is possible if Conditions (G5) and (G6) are satisfied) and by making $a_{0}= \pm 1$ for $k=2$ (provided that (G5) holds). Thus, we get the following two propositions.

Proposition 15 Let $D \in \mathcal{A}_{0}(2 k+2,2 k), k>2$ satisfy non-degeneracy Conditions ( $\left.\mathrm{G} 1\right)$, $(\mathrm{G} 2)$, and (G4)-(G6). Then, there exists a unique system of cogenerators $\widehat{\mathcal{K}}=\left(\hat{\omega}_{0}, \hat{\omega}_{1}\right)$ such that

$$
R_{\widehat{\mathcal{K}}}(t)=t^{k}+a_{k-2} t^{k-2}+a_{k-3} t^{k-3}+\cdots+a_{2} t^{2}+a_{1} t^{1}+a_{1}, \quad a_{1} \neq 0
$$

(there is no term of degree $k-1$ and the free term is equal to the coefficient standing in front of $\left.t^{1}\right)$.

Proposition 16 Let $D \in \mathcal{A}_{0}(6,4)$ satisfy Conditions (G1) and (G2) together with Conditions (G4) and (G5). Then, there exist exactly two systems of cogenerators, $\widehat{\mathcal{K}}_{1}$ and $\widehat{\mathcal{K}}_{2}$, such that

$$
R_{\widehat{\mathcal{K}}_{1}}(t)=R_{\widehat{\mathcal{K}}_{2}}(t)=t^{2} \pm 1
$$

These systems are of the form:

$$
\widehat{\mathcal{K}}_{1}, \widehat{\mathcal{K}}_{2}=\left(\hat{\omega}_{0}, \hat{\omega}_{0} \pm \underline{\hat{\omega}}\right),
$$

where $\underline{\hat{\hat{\omega}}}$ annihilates $D$ (the choice of $\pm \underline{\hat{\hat{\omega}}}$ does not affect the sign of the free term of $R_{\widehat{\mathcal{K}}_{1}}$ ).

Remark 17 Under the assumptions of Proposition 16, Condition (G6) automatically fails since every polynomial of degree 2 attains its extremum at the arithmetic mean of its roots.

Corollary 18 If the assumptions of Proposition 15 are satisfied, then the coefficients of the polynomial (25):

$$
\hat{a}_{1}, \hat{a}_{2}, \ldots, \hat{a}_{k-2}
$$

are canonically determined by the distribution.

Now, let us give an example of a distribution $D \in \mathcal{A}_{0}(2 k+2,2 k)$ satisfying Conditions (G1)-(G6) for $k>2$ and Conditions (G1)-(G5) for $k=2$.

Example 3 Let $n=2 k+2$ and $\mathcal{K}=\left(\omega_{1}, \omega_{2}\right)$,

$$
\begin{aligned}
\omega_{1} & =d x_{1}+d x_{2}+d x_{4}+\cdots+d x_{n}+x_{2} d x_{3}+x_{4} d x_{5}+\cdots+x_{n-2} d x_{n-1} \\
\omega_{2} & =d x_{2}+d x_{4}+\cdots+d x_{n}+x_{3} d x_{4}+x_{5} d x_{6}+\cdots+x_{n-3} d x_{n-2}+2 x_{n-1} d x_{n}+ \\
& +x_{n}\left(x_{2} d x_{4}+x_{4} d x_{6}+\cdots+x_{n-2} d x_{n}\right) .
\end{aligned}
$$


The above cogenerators differ from that given in Section 4 by the underlined coefficient 2 (in place of 1). We may repeat computations from that section to verify that Conditions (G1)(G2) are satisfied and

$$
\begin{array}{ll}
Y_{0}(0) & =-\frac{\partial}{\partial x_{2}}, \quad Y_{1}(0)=-\frac{\partial}{\partial x_{4}}, \quad \ldots, \quad Y_{k}(0)=-\frac{\partial}{\partial x_{n}} \\
{\left[Y_{k}, Y_{i}\right](0)} & =-\frac{\partial}{\partial x_{2 i+3}}, \quad \text { for } i=0,1, \ldots, k-2, \\
{\left[Y_{k}, Y_{k-1}\right](0)} & =-\frac{1}{2} \frac{\partial}{\partial x_{n-1}} .
\end{array}
$$

Condition (G3) is satisfied, since

$$
\operatorname{dim}\left(C_{0}^{D}+\left[C^{D}, C^{D}\right]_{0}\right) \geq 2 k
$$

and $C_{0}^{D}+\left[C^{D}, C^{D}\right]_{0} \subset D_{0}$. As in Example 2, we compute the polynomial $R_{\mathcal{K}}$ (at the origin), up to a factor making this polynomial monic:

$$
R_{\mathcal{K}}(t)=\operatorname{det}\left(t R_{\omega_{2}}+(1-t) R_{\omega_{1}}\right)=-\frac{1}{2}(1-t)^{k}+\sum_{i=0}^{k}(1-t)^{i} t^{k-i} .
$$

The coefficient of the term $t^{k}$ equals

$$
a_{k}=\frac{1}{2}(-1)^{k+1}+\sum_{i=0}^{k}(-1)^{i}=\frac{1}{2},
$$

and that of term $t^{k-1}$ :

$$
a_{k-1}=\frac{1}{2} k(-1)^{k}+\sum_{i=0}^{k} i(-1)^{i-1}= \begin{cases}0, & \text { if } k \text { is even, } \\ \frac{1}{2}, & \text { if } k \text { is odd. }\end{cases}
$$

Thus, for an even $k$, the arithmetic mean of the roots of $R_{\mathcal{K}}$ equals

$$
\bar{t}=-\frac{a_{k-1}}{k a_{k}}=0 \text {. }
$$

Since

$$
R_{\mathcal{K}}(0)=\frac{1}{2} \quad \text { and } \quad R_{\mathcal{K}}^{\prime}(0)=\frac{2-k}{2},
$$

the polynomial $R_{\mathcal{K}}$ satisfies Conditions (G4)-(G6) for every even $k>2$ (for $k=2$ Condition (G6) fails). In order to show that the same holds for odd $k>1$, we observe that for such a $k$ :

and (cf. (26))

$$
\bar{t}=-\frac{a_{k-1}}{k a_{k}}=-\frac{1}{k}
$$

$$
R_{\mathcal{K}}(\bar{t})=\frac{1}{2}(1-\bar{t})^{k}+\sum_{i=0}^{k-1}(1-\bar{t})^{i} \bar{t}^{k-i}>0 .
$$

It remains to show that $R_{\mathcal{K}}^{\prime}(\bar{t}) \neq 0.1$ By direct computation, we get (for odd $k$ ):

$$
k^{k-2} R_{\mathcal{K}}^{\prime}\left(\frac{-1}{k}\right)=-\frac{k^{2}(1+k)^{k-1}-2(1+k)^{k-1}-2 k-6}{2(2+k)^{2}} .
$$

Thus, $R_{\mathcal{K}}^{\prime}(\bar{t})<0$, since for odd $k \geq 3$,

$$
k^{2}(1+k)^{k-1}-2(1+k)^{k-1} \geq 7(1+k)^{k-1} \geq 7(1+k)^{2}>2 k+6 .
$$




\subsection{Canonical Generators of $C^{D}$}

Let a distribution $D \in \mathcal{A}_{q}(2 k+2,2 k)$ has affine cogenerators $\widehat{\mathcal{K}}=\left(\hat{\omega}_{0}, \hat{\omega}_{1}\right)$ introduced in Proposition 15 and 16 for $k>2$ and $k=2$, respectively. System $\widehat{\mathcal{K}}$ defines a parametrization of the fiber $S_{q}^{D}$ :

$$
t \mapsto \omega=t \hat{\omega}_{1}+(1-t) \hat{\omega}_{0} \mapsto \Theta(t)=X_{\omega},
$$

defined for all $t$ at which $R_{\widehat{\mathcal{K}}}(t) \neq 0$ (vector field $X_{\omega}$ is defined after the formulation of Proposition 8). The mapping

$$
\Phi: t \mapsto R_{\widehat{\mathcal{K}}}(t)(\Theta(t)-\Theta(0)) \in \underline{C}^{D}
$$

is polynomial. Since the image of $\Theta$ must be $(k+1)$-dimensional, we thus have the following sequence of linearly independent vectors:

$$
\hat{f}_{0}=\Theta(0) \in S_{q}^{D}, \quad \hat{f}_{i}=\frac{\Phi^{(i)}(0)}{i !} \in \underline{C}_{q}^{D}, \quad i=1,2, \ldots,
$$

Since the construction of these vectors depends smoothly on the point $q$, we may write about smooth vector fields $\hat{f}_{i}, i=0,1, \ldots$ defined in a neighborhood of that point. The construction of these vector fields implies that the mapping $\Theta$ gets the form presented in Theorems 3 and 4:

$$
\Theta(t)=\hat{f_{0}}+\frac{1}{\widehat{R}(t)} \sum_{i=1}^{k} t^{i} \hat{f}_{i} .
$$

Acknowledgments Some of the results presented in the paper are excerpted from the PhD thesis [19]. The author would like to express his gratitude to professor B. Jakubczyk, the dissertation adviser, whose kind support and constructive criticism had an important impact on the final form of this work.

The author would also thank the anonymous reviewer, whose exhaustive remarks and suggestions have enabled to improve and shorten the material presented in the paper.

Open Access This article is distributed under the terms of the Creative Commons Attribution License which permits any use, distribution, and reproduction in any medium, provided the original author(s) and the source are credited.

\section{References}

1. Agrachev AA. Feedback-invariant optimal control theory and differential geometry. II. Jacobi curves for singular extremals. J Dynam Control Syst 1998;4(4):583-604. MR1662927 (2000a:49007).

2. Agrachev A, Zelenko I. On feedback classification of control-affine systems with one- and two-dimensional inputs. SIAM J Control Optim 2007;46(4):1431-1460. (electronic) MR2346387 (2009e:93037).

3. Bonnard B, Chyba M. Singular trajectories and their role in control theory, Mathématiques \& Applications (Berlin) [Mathematics \& Applications], vol. 40. Berlin: Springer-Verlag; 2003. MR1996448 (2004f:93001).

4. Bismut J. Large deviations and the Malliavin calculus, Progress in Mathematics, vol. 45. Boston, MA: Birkhäuser Boston Inc; 1984. MR755001 (86f:58150).

5. Bonnard B. Feedback equivalence for nonlinear systems and the time optimal control problem. SIAM J Control Optim 1991;29(6):1300-1321. MR1132184 (93b:93030).

6. Chitour Y, Jean F, Trélat E. Singular trajectories of driftless and control-affine systems, Proceedings of Decision and Control, 2005 and 2005 European Control Conference; 2005, pp. 940-944. 
7. Chitour Y, Jean F, Trélat E. Genericity results for singular curves. J Diff Geom 2006;73(1):4573. MR2217519 (2007h:58006).

8. Chitour Y, Jean F, Trélat E. Singular trajectories of control-affine systems. SIAM J Control Optim 2008;47(2):1078-1095. MR2385874 (2009b:93049).

9. Gantmacher FR. 1959. Applications of the theory of matrices, Translated by J. L. Brenner, with the assistance of D. W. Bushaw and S. Evanusa, Interscience Publishers, Inc. MR0107648 (21 \#6372b).

10. Gauger M. A. On the classification of metabelian Lie algebras. Trans Amer Math Soc 1973;179:293-329. 0325719 (48 \#4066).

11. Hsu L. Calculus of variations via the Griffiths formalism. J Diff Geom 1992;36(3):551-589. MR1189496 (94a:58003).

12. Jakubczyk B. Equivalence and invariants of nonlinear control systems, Nonlinear controllability and optimal control. Monogr Textbooks Pure Appl Math 1990;133:177-218. MR1061386 (91c:93045).

13. Jakubczyk B, Kryński W, Pelletier F. Characteristic vector fields of generic distributions of corank 2. Ann Inst H Poincaré Anal Non Linéaire 2009;26(1):23-38. MR2483811 (2010f:58004).

14. Jakubczyk B, Respondek W. Feedback classification of analytic control systems in the plane, Analysis of controlled dynamical systems (Lyon, 1990), Progr. Systems Control Theory, vol. 8. Boston, MA: Birkhäuser Boston; 1991, pp. 263-273. MR1132000 (93b:93027).

15. Jakubczyk B, Zhitomirskii M. Distributions of corank 1 and their characteristic vector fields. Trans Amer Math Soc 2003;355(7):2857-2883. (electronic) MR1975403 (2004c:58006).

16. Montgomery R. A survey of singular curves in sub-Riemannian geometry. J ynam Control Syst 1995;1(1):49-90. MR1319057 (95m:53060).

17. Montgomery R. A tour of subriemannian geometries, their geodesics and applications, Mathematical Surveys and Monographs, vol. 91, American Mathematical Society, Providence, RI; 2002. MR1867362 (2002m:53045).

18. Pontryagin LS, Boltyanskii VG, Gamkrelidze RV, Mishchenko EF. 1964. The mathematical theory of optimal processes, Translated by D. E. Brown, A Pergamon Press Book. The Macmillan Co., New York. MR0186436 (32 \#3896).

19. Rupniewski MW. Geometry of even rank affine distributions and their singular curves, Ph.D. thesis, Institute of Mathematics, Polish Academy of Sciences. in Polish; 2008.

20. Respondek W, Zhitomirskii M. Feedback classification of nonlinear control systems on 3-manifolds. Math Control Signals Syst 1995;8(4):299-333. MR1403291 (97i:93051). 